\title{
Electronic Nose Technology Based on Quantum Dot Filters
}

\author{
Zhenan Li*, Jie Bao \\ Department of Electronic Engineering, Tsinghua University, Beijing, China \\ Email: *lizn14@mails.tsinghua.edu.cn
}

How to cite this paper: Li, Z.N. and Bao, J. (2017) Electronic Nose Technology Based on Quantum Dot Filters. Optics and Photonics Journal, 7, 26-32.

https://doi.org/10.4236/opj.2017.78B005

Received: April 26, 2017

Accepted: August 7, 2017

Published: August 10, 2017

\begin{abstract}
The electronic nose with chemical dyes as sensor can react with target gas and have specific color changes. In general, RGB camera collects a group of images to record these changes used for pattern recognition. RGB filters are not sensitive to the slight color changes, which limits the performance of this kind of electronic nose. This paper demonstrates using quantum dot spectroscopy technology to solve this problem. Multiple quantum dot filters are placed on the surface of image sensor. When capturing images, there are more response channels of the same incident light than RGB filters. Simulation and experiment both prove that quantum dot filters with appropriate processing are more sensitive to color changes than RGB filters.
\end{abstract}

\section{Keywords}

Quantum Dots, Spectrum, RGB Filters, Pattern Recognition

\section{Introduction}

An electronic nose is an equipment that imitates the olfactory system of mammals. In general, it consists of two parts. One is an array of gas sensors acting like olfactory receptor cells of creatures, which can convert the gas information to easy detected variables, such as voltage, resistance. The other is a pattern recognition system to recognize the type, concentration or acidity of target gas according to the measured variables like creature brains [1] [2] [3]. There is a kind of sensor array made of specific chemical dyes, which can react with target gas and change colors. RGB camera collects a group of images to record these changes and recognition system output the results through image processing [4] [5] [6]. The limitation of this method is that RGB camera is not sensitive to the variety of reflection spectrum of chemical dyes before and after the reaction. 
Sometimes, RGB camera can even get same response with different reflection spectrum (metamerism).

Quantum dot spectroscopy is a kind of emerging technology, which use a variety of quantum dot colloids to make films as filters. Films with different light transmission curves filter the incident light spectrum. The image sensor can record the transmitted intensities of different filters simultaneously. The origin spectrum can be reconstructed by resolving a group of filter transmission equations [7].

This paper demonstrates using a group of quantum dot filters to capture the slight color changes of electronic nose sensors and trying to get better results than RGB filters by appropriately processing on the quantum dot data.

\section{Experiment Design}

In the experiment, acetic acid with different concentrations generates target gas by volatilization. Specific sensors made of $\mathrm{pH}$ indicator Bromothymol Green (with $\mathrm{NaOH}$ solution) react with target gas in a closed container for a certain time. The changes before and after the reaction are measured by quantum dot camera, color camera and spectrometer respectively. Quantum dot camera, which has nine filters on the image sensor surface, captures the transmitted intensity of signal light. Color camera with RGB filters on the surface of the image sensor, collects data for comparison. Spectrometer records reflection spectrum of sensors for simulation.

For data processing, acetic acid with five different concentrations $(0.0 \%, 0.6 \%$, $1.2 \%, 1.8 \%$ and $2.4 \%$ ) correspond to class labels $1-5$. Thus, pattern recognition method can be used for quantitative analysis. The recognition rate, false alarm rate can be used to judge the performance of RGB filters and quantum dot filters.

Spectrum data can simulate the outputs of RGB filters and quantum dot filters. In the simulation, it is convenient to find appropriate method for processing quantum dot data with the help of spectrum. Finally, actual data is used to verify the simulation and differences are analyzed.

\section{Simulation}

Regardless of the quantum efficiency of image sensor, the transmitted intensities of RGB filters and quantum dot filters are described as Equation (1)

$$
I_{i}=\sum_{\lambda} \Phi(\lambda) T_{i}(\lambda), i=r, g, b, q 1, q 2, \ldots, q 9
$$

where $\Phi(\lambda)$ is the incident light spectrum, $T_{i}(\lambda)$ is the transmission curve of different filters, and $I_{i}$ is the transmitted intensity corresponding to $T_{i}(\lambda)$. The spectrum data measured by spectrometer perform as incident light. Outputs are the projection of incident light under the RGB and quantum dot, whose transmission curves shows in Figure 1. 


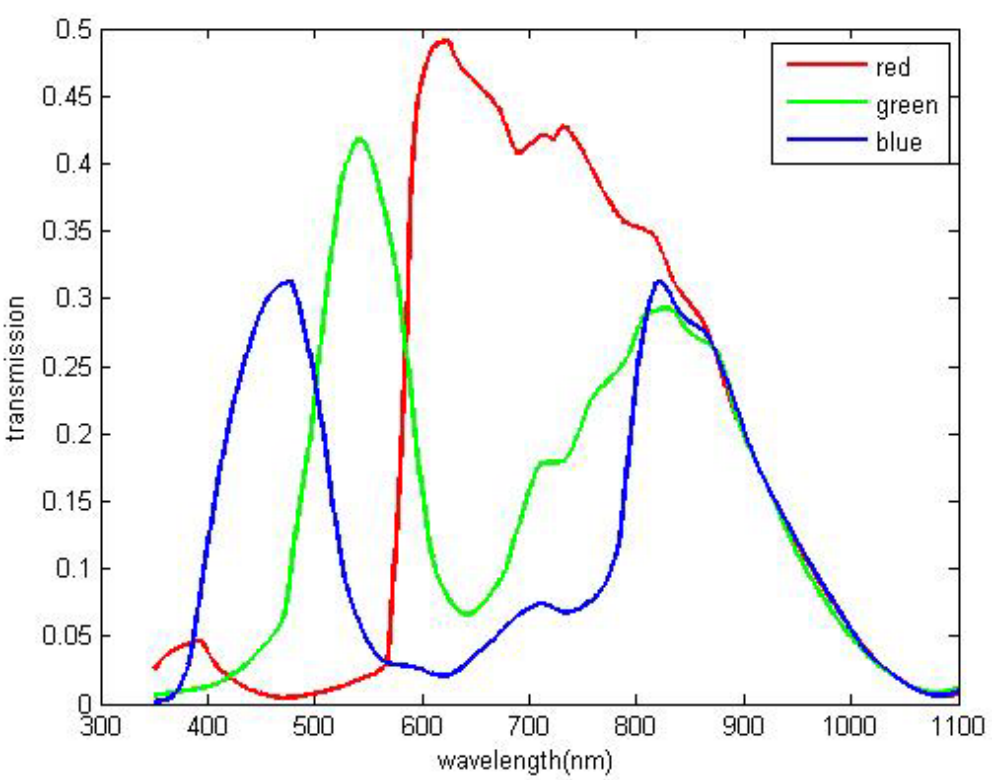

(a)
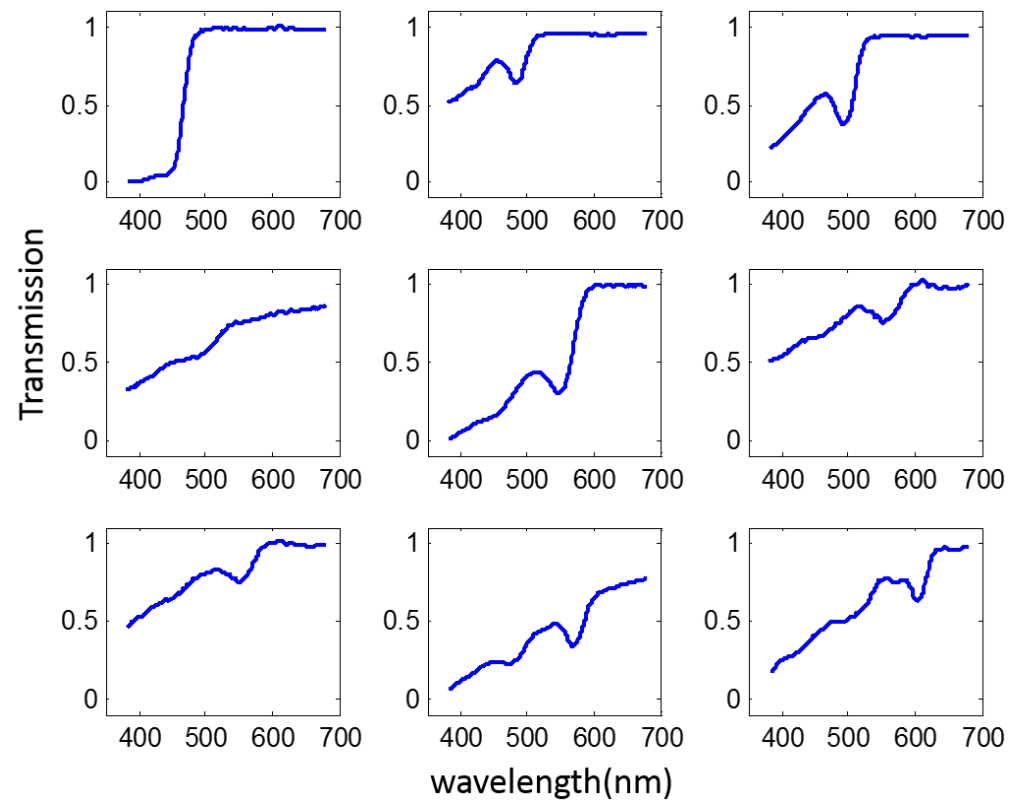

(b)

Figure 1. RGB (a) and quantum dot (b) transmission curves.

\subsection{Preprocessing and Recognition}

As the origin state of different groups is not exactly the same, changes of the reaction cannot be shown simply by after-reaction data. Therefore, feature vectors are demanded to represent the changes. The ratio of the intensity is capable for feature vector.

$$
\begin{gathered}
R_{s}=\left(I_{\lambda 1} / I_{\lambda 1}^{0}, I_{\lambda 2} / I_{\lambda 2}^{0}, \ldots, I_{\lambda n} / I_{\lambda n}^{0}\right) \\
R_{r g b}=\left(I_{r} / I_{r}^{0}, I_{g} / I_{g}^{0}, I_{b} / I_{b}^{0}\right)
\end{gathered}
$$




$$
R_{q d}=\left(I_{q 1} / I_{q 1}^{0}, I_{q 2} / I_{q 2}^{0}, \ldots, I_{q 9} / I_{q 9}^{0}\right)
$$

$R_{s}, R_{r g b}$ and $R_{q d}$ are feature vectors of spectrum, RGB and quantum dot. As in Equation (2), spectrum is discrete from $360 \mathrm{~nm}-700 \mathrm{~nm}$ with $1 \mathrm{~nm}$ interval. Support vector machine (SVM) is used to train and test feature vectors [8]. Table 1 shows the results of the overall recognition rate and false alarm rate of five different concentrations.

Spectrum has the best results due to high dimension and completeness of target wavelength band. Direct projection of spectrum under RGB and quantum dot base result bad for not sensitive to color changes. Delicate sensitive base is needed to achieve better result, which can be devised using existing quantum dot base.

Down sampling is equivalent to a group of filters with square band-pass curves whose bandwidth is $n \mathrm{~nm}$. Spectrum does an average every $n \mathrm{~nm}$. The amount of data becomes $1 / n$ of the original. Table 2 shows the classification results of different compressed rate. It is feasible to design band-pass base with quantum dot for improving the performance.

\subsection{Quantum Dot Processing}

Quantum dot filters are used to fit band-pass filter. Target transmission base is a series of Gaussian band pass curves. The center wavelength of these curves covers from $400 \mathrm{~nm}-640 \mathrm{~nm}$ with $20 \mathrm{~nm}$ interval and full width at half maximum is $20 \mathrm{~nm}$. As in Equation (5), $T_{a}(\lambda)$ represents the target curves and $I_{a}$ is the trans-mitted intensity.

$$
I_{a}=\sum_{\lambda} \Phi(\lambda) T_{a}(\lambda)
$$

The quantum dot base can generate new base by linear combination.

$$
\tilde{T}_{a}(\lambda)=\sum_{i} a_{i} T_{i}(\lambda)
$$

New transmission base can be as close as possible to the target base by adjusting the linear coefficients. The optimization equation is to minimize the square error between target base and new base.

Table 1. Comparison of spectrum, RGB and quantum dot classification results.

\begin{tabular}{ccc}
\hline Feature Vector & Recognition Rate & False Alarm Rate \\
\hline$R_{s}$ & 0.8273 & 0.0423 \\
$R_{r g b}$ & 0.6263 & 0.0935 \\
$R_{q d}$ & 0.5501 & 0.1128 \\
\hline
\end{tabular}

Table 2. Classification results of compressed spectrum.

\begin{tabular}{ccccccc}
\hline Compression Rate & 1 & 4 & 10 & 20 & 68 & 85 \\
\hline Recognition Rate & 0.8273 & 0.8187 & 0.8540 & 0.8636 & 0.7511 & 0.7184 \\
\hline
\end{tabular}




$$
\min \left\|T_{a}(\lambda)-\tilde{T}_{a}(\lambda)\right\|^{2} \text {, s.t. } 0 \leq \tilde{T}_{a}(\lambda) \leq 1
$$

Simplified solution is Equation (8).

$$
\boldsymbol{a}=\arg \min \left\|T_{a}(\lambda)-\tilde{T}_{a}(\lambda)\right\|^{2}=\arg \min \left\|T_{a}(\lambda)-\sum_{i} a_{i} T_{i}(\lambda)\right\|^{2}
$$

The outputs of reconstructed target base can be generated by linear combination with coefficients $\boldsymbol{a}$.

$$
\tilde{I}_{a}=\sum_{\lambda} \Phi(\lambda) \tilde{T}_{a}(\lambda)=\sum_{\lambda} \Phi(\lambda)\left(\sum_{i} a_{i} T_{i}(\lambda)\right)=\sum_{i} a_{i}\left(\sum_{\lambda} \Phi(\lambda) T_{i}(\lambda)\right)=\sum_{i} a_{i} I_{i}
$$

New feature vector $R_{a}$ is as Equation (10).

$$
\tilde{R}_{a}=\left(\tilde{I}_{a 1} / \tilde{I}_{a 1}^{0}, \tilde{I}_{a 2} / \tilde{I}_{a 2}^{0}, \ldots, \tilde{I}_{a 12} / \tilde{I}_{a 12}^{0}\right)
$$

The target base and reconstructed base are shown in Figure 2. Most reconstructed base fit well on target base. While for spectrum below $460 \mathrm{~nm}$ and above $620 \mathrm{~nm}$, quantum dot base has similar shape and linear combination cannot perform well. Thus, the Gaussian band-pass base with center wavelength below $460 \mathrm{~nm}$ and above $620 \mathrm{~nm}$ have high reconstruction errors. Table 3 shows the improvement of quantum dot data after processing.

\section{Verification}

Table 4 shows the actual data captured by RGB camera and quantum dot camera. The classification results have the same trend as the simulation results. Differently, processed quantum dot outputs do not have as much improvement as the simulation process. The digital accuracy and acquire noise of camera affect the results.

Table 3. Comparison of simulation.

\begin{tabular}{ccc}
\hline Feature Vector & Recognition Rate & False Alarm Rate \\
\hline$R_{r g b}$ & 0.6263 & 0.0935 \\
$R_{q d}$ & 0.5501 & 0.1128 \\
$\tilde{R}_{a}$ & 0.7702 & 0.0583 \\
\hline
\end{tabular}

Table 4. Comparison of actual data classification.

\begin{tabular}{ccc}
\hline Feature Vector & Recognition Rate & False Alarm Rate \\
\hline$R_{r g b}$ & 0.6351 & 0.0912 \\
$R_{q d}$ & 0.6222 & 0.0944 \\
$\tilde{R}_{a}$ & 0.7111 & 0.0722 \\
\hline
\end{tabular}



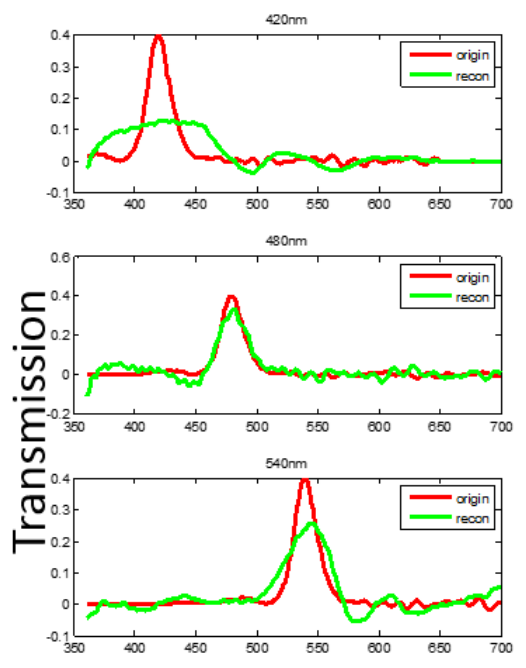

$600 \mathrm{~nm}$

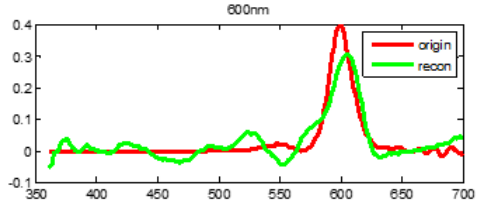

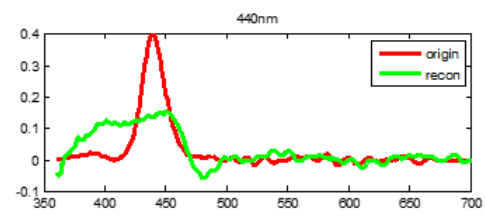

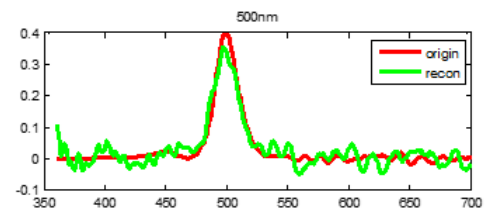

$500 \mathrm{~nm}$

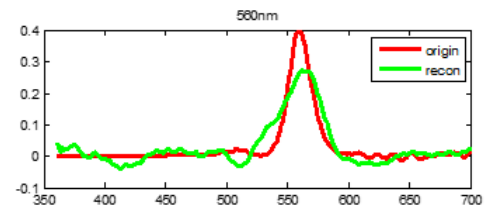

$620 \mathrm{~nm}$

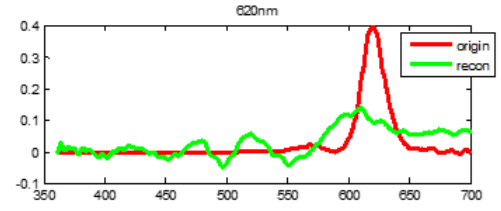

Wavelength(nm)
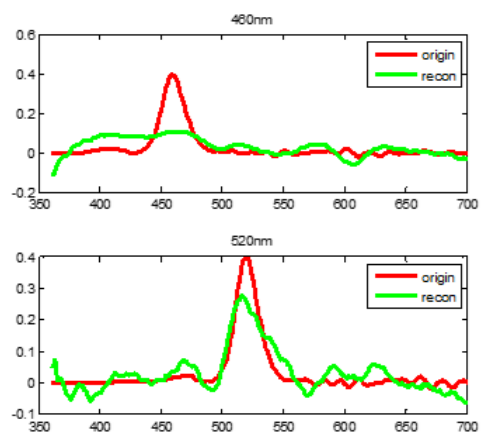

$30 \mathrm{~nm}$

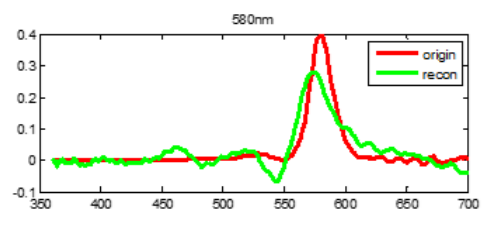

$640 \mathrm{~nm}$

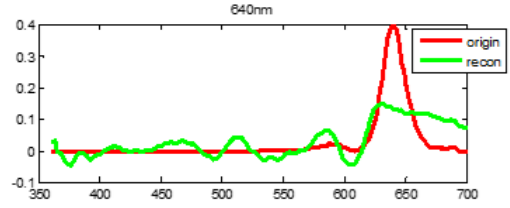

Figure 2. Target base and reconstructed base by quantum dot base.

\section{Conclusion}

Quantum dot filters are more sensitive to slight color changes and have a higher dimension compared to RGB filters. With appropriate data processing method, the information of a certain wavelength band can be enlarged. If the number of quantum dots is enough, the origin spectrum can be even resolved and used for classification. Furthermore, quantum dot base has larger area of integration than RGB transmission base so weak light can be captured.

\section{References}

[1] Gardner, J.W. and Bartlett, P.N. (1994) A Brief History of Electronic Noses. Sensors \& Actuators B Chemical, 18-19, 210-211. https://doi.org/10.1016/0925-4005(94)87085-3

[2] Wilson, A.D. and Baietto, M. (2009) Applications and Advances in Electronic-Nose Technologies. Sensors, 9, 5099-5148. https://doi.org/10.3390/s90705099

[3] Tang, K.T., Chiu, S.W., Pan, C.H., Hsieh, H.Y., Liang, Y.S. and Liu, S.C. (2010) Development of a Portable Electronic Nose System for the Detection and Classification of Fruity Odors. Sensors, 10, 9179-9193. https://doi.org/10.3390/s101009179

[4] Rakow, N.A. and Suslick, K.S. (2000) A Colorimetric Sensor Array for Odour Visualization. Nature, 406, 710-713. https://doi.org/10.1038/35021028

[5] Suslick, K.S. (2004) An Optoelectronic Nose: “Seeing” Smells by Means of Colorimetric Sensor Arrays. MRS Bulletin, 29, 720-725. https://doi.org/10.1557/mrs2004.209

[6] Sen, A., Albarella, J.D., Carey, J.R., Kim, P. and Iii, M.N. (2008) Low-Cost Colorimetric Sensor for the Quantitative Detection of Gaseous Hydrogen Sulfide. Sensors 
\& Actuators B Chemical, 134, 234-237. https://doi.org/10.1016/j.snb.2008.04.046

[7] Bao, J. and Bawendi, M.G. (2015) A Colloidal Quantum Dot Spectrometer. Nature, 523, 67-70. https://doi.org/10.1038/nature14576

[8] Liang, W., Zhang, L.N., Li, X.W. and Zuo, Y.D. (2012) Research of Electronic Nose Pattern Recognition Algorithm Based on Svm. Applied Mechanics \& Materials, 220-223, 2244-2247.

https://doi.org/10.4028/www.scientific.net/AMM.220-223.2244

Submit or recommend next manuscript to SCIRP and we will provide best service for you:

Accepting pre-submission inquiries through Email, Facebook, LinkedIn, Twitter, etc. A wide selection of journals (inclusive of 9 subjects, more than 200 journals)

Providing 24-hour high-quality service

User-friendly online submission system

Fair and swift peer-review system

Efficient typesetting and proofreading procedure

Display of the result of downloads and visits, as well as the number of cited articles Maximum dissemination of your research work

Submit your manuscript at: http://papersubmission.scirp.org/

Or contact opj@scirp.org 